\title{
String theoretic QCD axions in the light of PLANCK and BICEP2
}

\author{
Kiwoon Choi, ${ }^{a, b}$ Kwang Sik Jeong ${ }^{c}$ and Min-Seok Seo ${ }^{a}$ \\ ${ }^{a}$ Center for Theoretical Physics of the Universe, IBS, \\ 70 Yuseong-daero 1689-gil, Yuseong-gu, Daejeon 305-811, Korea \\ ${ }^{b}$ Department of Physics, KAIST, \\ 291 Daehak-ro, Yuseong-gu, Daejeon 305-701, Korea \\ ${ }^{c} D E S Y$, \\ Notkestrasse 85, 22607 Hamburg, Germany \\ E-mail: kchoi@kaist.ac.kr, kwangsik.jeong@desy.de, \\ minseokseo@ibs.re.kr
}

ABSTRACT: The QCD axion solving the strong CP problem may originate from antisymmetric tensor gauge fields in compactified string theory, with a decay constant around the GUT scale. Such possibility appears to be ruled out now by the detection of tensor modes by BICEP2 and the PLANCK constraints on isocurvature density perturbations. A more interesting and still viable possibility is that the string theoretic QCD axion is charged under an anomalous $\mathrm{U}(1)_{A}$ gauge symmetry. In such case, the axion decay constant can be much lower than the GUT scale if moduli are stabilized near the point of vanishing Fayet-Illiopoulos term, and $\mathrm{U}(1)_{A}$-charged matter fields get a vacuum value $v \sim\left(m_{\mathrm{SUSY}} M_{P l}^{n}\right)^{1 /(n+1)}(n \geq 0)$ induced by a tachyonic SUSY breaking mass $m_{\mathrm{SUSY}}$. We examine the symmetry breaking pattern of such models during the inflationary epoch with $H_{I} \simeq 10^{14} \mathrm{GeV}$, and identify the range of the QCD axion decay constant, as well as the corresponding relic axion abundance, consistent with known cosmological constraints. In addition to the case that the PQ symmetry is restored during inflation, i.e. $v\left(t_{I}\right)=0$, there are other viable scenarios, including that the $\mathrm{PQ}$ symmetry is broken during inflation with $v\left(t_{I}\right) \sim\left(4 \pi H_{I} M_{P l}^{n}\right)^{1 /(n+1)} \sim 10^{16}-10^{17} \mathrm{GeV}$ due to the Hubble-induced $D$-term $D_{A} \sim 8 \pi^{2} H_{I}^{2}$, while $v\left(t_{0}\right) \sim\left(m_{\mathrm{SUSY}} M_{P l}^{n}\right)^{1 /(n+1)} \sim 10^{9}-5 \times 10^{13} \mathrm{GeV}$ in the present universe, where $v\left(t_{0}\right)$ above $10^{12} \mathrm{GeV}$ requires a fine-tuning of the axion misalignment angle. We also discuss the implications of our results for the size of SUSY breaking soft masses.

KEYwords: Cosmology of Theories beyond the SM, Compactification and String Models ArXiv EPRINT: 1404.3880 


\section{Contents}

1 Introduction and summary $\quad 1$

2 String theoretic QCD axion $\quad 4$

3 Cosmological constraints $\quad 9$

4 Axion decay constant during and after inflation $\quad 15$

$\begin{array}{llr}5 & \text { Conclusions } & 20\end{array}$

\section{Introduction and summary}

The strong CP problem ${ }^{1}$ of the Standard Model of particle physics is about the question why the strong $\mathrm{CP}$ violating parameter $\bar{\theta}=\theta_{\mathrm{QCD}}+\arg \left(y_{u} y_{d}\right)$ is smaller than $10^{-10}$, while the weak CP violating Kobayashi-Maskawa phase originating from the same quark Yukawa couplings $y_{u, d}$ is of order unity. Presently the most compelling solution to this problem is to introduce a non-linearly realized anomalous global U(1) symmetry, the Peccei-Quinn (PQ) symmetry $[4,5]$, which predicts a pseudo-Goldstone boson, the QCD axion, whose vacuum expectation value (VEV) can be identified as $\bar{\theta}$ [6-11]. Yet, there still remain some questions. One question is, what is the origin of the PQ symmetry? The PQ symmetry is required to be explicitly broken by the QCD anomaly, while being protected well from other forms of explicit breaking. In view of that global symmetry is not respected in general by UV physics at scales where quantum gravity becomes important [12-15], the existence of such global symmetry at low energy scales may require a specific form of UV completion of the model [16-18]. Another question is about the mechanism to determine the axion decay constant $f_{a}$, which determines most of the phenomenological consequences of the QCD axion, including the cosmological ones.

It has been known for many years that string theory provides an attractive theoretical framework to address these questions [19]. String theory includes a variety of higherdimensional antisymmetric tensor gauge fields, whose zero modes behave like axions in the 4-dimensional effective theory. The shift symmetries associated with these axion-like fields are valid in perturbation theory [20,21]. It is then conceivable that a certain combination of the shift symmetries is broken dominantly by the QCD anomaly, and therefore can be identified as the PQ symmetry solving the strong CP problem. As for the decay constant, if the compactification scale is comparable to the Planck scale, the decay constants of such stringy axions are estimated to be [22-26],

$$
f_{a} \sim g^{2} M_{P l} / 8 \pi^{2},
$$

\footnotetext{
${ }^{1}$ For a recent review, see, e.g., refs. [1-3].
} 
where the factor $8 \pi^{2}$ comes from the convention for the axion decay constant, and $M_{P l} \simeq$ $2.4 \times 10^{18} \mathrm{GeV}$ is the reduced Planck scale. Although it is subject to severe cosmological constraints [27-37], such QCD axion arising from antisymmetric tensor gauge fields in compactified string theory has been considered to be a viable possibility for many years.

An interesting generalization of this scheme, involving an anomalous $\mathrm{U}(1)_{A}$ gauge symmetry with a nonzero $\mathrm{U}(1)_{A}$ - $\mathrm{SU}(3)_{c}-\mathrm{SU}(3)_{c}$ anomaly cancelled by the 4-dimensional Green-Schwarz (GS) mechanism [38], has been discussed before for the purpose of having an intermediate scale QCD axion even when the compactification scale is comparable to the Planck scale $[24,39-42]$. It is based on the compactification models in which moduli are stabilized at the point of vanishing $\mathrm{U}(1)_{A}$ Fayet-Illiopoulos $(\mathrm{FI})$ term $\xi_{\mathrm{FI}}=0$ in the supersymmetric limit, when all $\mathrm{U}(1)_{A}$-charged matter fields $\phi$ are set to zero. Such supersymmetric solutions are known to exist in many of the Type II string theory with $D$-branes [21, 43-47], as well as in the heterotic string theory with $\mathrm{U}(1)$ gauge bundles [4850]. In the limit of $\xi_{\mathrm{FI}}=\phi=0$, the $\mathrm{U}(1)_{A}$ gauge boson obtains a superheavy mass $M_{A} \sim M_{P l} / 8 \pi^{2}$ by absorbing the stringy axion $\theta_{\text {st }}$ implementing the GS anomaly cancellation mechanism, while leaving an unbroken perturbative global U(1) symmetry, which corresponds the global part of $\mathrm{U}(1)_{A}$ without the transformation of $\theta_{\mathrm{st}}$. By construction, this perturbative global $\mathrm{U}(1)$ symmetry has nonzero $\mathrm{U}(1)-\mathrm{SU}(3)_{c}-\mathrm{SU}(3)_{c}$ anomaly, and therefore can be identified as the PQ symmetry solving the strong CP problem.

To satisfy the astrophysical constraints on the QCD axion, this PQ symmetry should be spontaneously broken at a scale higher than $10^{9} \mathrm{GeV}[1-3]$. For this, some $\mathrm{U}(1)_{A^{-}}$ charged matter field $\phi$ should have a tachyonic supersymmetry (SUSY) breaking scalar mass $m_{\mathrm{SUSY}}$, destabilizing the supersymmetric solution $\xi_{\mathrm{FI}}=\phi=0$. The matter scalar field $\phi$ then takes a vacuum value $\langle\phi\rangle>10^{9} \mathrm{GeV}$ by an interplay between the tachyonic SUSY breaking mass term and a supersymmetric higher order term which schematically takes the form $|\phi|^{2 n+4} / M_{P l}^{2 n}$ with $n \geq 0$ if the cutoff-scale of the model is assumed to be comparable to the Planck scale $[51,52]$. This scheme to determine $\langle\phi\rangle$ leads to an appealing connection between the axion scale and the SUSY breaking scale as

$$
f_{a} \simeq\langle\phi\rangle \sim\left(m_{\mathrm{SUSY}} M_{P l}^{n}\right)^{1 /(n+1)} \quad(n \geq 0),
$$

which makes it possible that a wide range of the QCD axion decay constant much lower than the Planck scale is obtained within the framework of string theory.

The recent detection of tensor modes in the cosmic microwave background (CMB) by BICEP2 [53] has important implications for axion cosmology [54-56], particularly for the string theoretic QCD axion. First of all, the BICEP2 results imply that the inflation energy scale is about $10^{16} \mathrm{GeV}$. This suggests that the string compactification scale is higher than $10^{16} \mathrm{GeV}$, and therefore the estimate (1.1) of the decay constants of stringy axion-like fields is at least qualitatively correct. For the expansion rate $H_{I} \sim 10^{14} \mathrm{GeV}$, if the PQ symmetry were spontaneously broken during inflation, the corresponding QCD axion is severely constrained by the PLANCK constraints on isocurvature density perturbations and nonGaussianity [57]. ${ }^{2}$ As we will see, this rules out the simple possibility that the QCD axion

\footnotetext{
${ }^{2}$ It is in principle possible that the axion under the consideration obtains a heavy mass $m_{a}\left(t_{I}\right) \gtrsim H_{I}$
} 
corresponds to a combination of the zero modes of antisymmetric tensor fields in compactified string theory, having a decay constant $f_{a} \sim g^{2} M_{P l} / 8 \pi^{2}$. On the other hand, in the presence of an anomalous $\mathrm{U}(1)_{A}$ gauge symmetry with vanishing FI term, under which the QCD axion is charged, the model can have rich symmetry breaking patterns during inflation, while giving a present axion decay constant much lower than $g^{2} M_{P l} / 8 \pi^{2}$. This may make it possible that the model allows a variety of different cosmologically viable scenarios.

In this paper, we examine the symmetry breaking pattern of the string theoretic QCD axion models involving an anomalous $\mathrm{U}(1)_{A}$ gauge symmetry during the inflationary epoch with $H_{I} \simeq 10^{14} \mathrm{GeV}$. We identify the allowed range of the axion decay constant in such models, as well as the corresponding relic axion abundance, being consistent with known cosmological constraints, within a general framework in which the axion scale during inflation can be different from the axion scale in the present universe. We note first that if the PQ symmetry were broken during inflation, the cosmological constraints can be satisfied only when the axion scale during inflation is much higher than the present axion scale. The most natural setup to realize this possibility is to generate the axion scale through SUSY breaking effects. We show that indeed the string theoretic QCD axion models with anomalous $\mathrm{U}(1)_{A}$ gauge symmetry provides such setup. If the modulus-axion superfield implementing the GS mechanism is not sequestered from the SUSY breaking by the inflaton sector, which would be the case in generic situations, $\mathrm{U}(1)_{A}$-charged matter fields develop a large expectation value during inflation,

$$
\left\langle\phi\left(t_{I}\right)\right\rangle \sim\left(\sqrt{8 \pi^{2}} H_{I} M_{P l}^{n}\right)^{1 /(n+1)},
$$

due to the tachyonic SUSY breaking scalar mass induced dominantly by the $\mathrm{U}(1)_{A} D$-term:

$$
m_{\phi}^{2}\left(t_{I}\right) \simeq q_{\phi} g_{A}^{2} D_{A}\left(t_{I}\right) \sim-8 \pi^{2} H_{I}^{2}
$$

while

$$
\left\langle\phi\left(t_{0}\right)\right\rangle \sim\left(m_{\mathrm{SUSY}} M_{P l}^{n}\right)^{1 /(n+1)},
$$

for the SUSY breaking scalar mass $m_{\text {SUSY }}$ in the present universe. Then the QCD axion during inflation has a much higher decay constant than the present value, and even is a different degree of freedom. As we will see, this makes it possible that a certain parameter space of the model is consistent with the constraints on isocurvature perturbations and non-Gaussianity, as summarized in figure 1 in section 3. The allowed range of the present axion decay constant for reasonable choice of model parameters is given by

$$
10^{9} \mathrm{GeV} \lesssim f_{a}\left(t_{0}\right) \lesssim 5 \times 10^{13} \mathrm{GeV}
$$

where $f\left(t_{0}\right) \gtrsim 10^{12} \mathrm{GeV}$ requires a fine-tuning of the axion misalignment angle as $\theta_{0} \lesssim$ $\mathcal{O}\left(10^{-1}\right)$. If we assume $\theta_{0}=\mathcal{O}(1)$, the allowed range is reduced to $f_{a}\left(t_{0}\right) \simeq 10^{9}-10^{11} \mathrm{GeV}$, with the axion dark matter making up roughly $0.1-10 \%$ of the total dark matter energy density.

during inflation, so is free from the isocurvature and non-Gaussianity constraints [58, 59]. However, it is not likely to be realized in our theoretical framework, as $m_{a}$ is protected by both the shift symmetry broken only by non-perturbative effects and the softly broken SUSY during inflation with $H_{I} \ll M_{P l}$. 
On the other hand, if the modulus-axion superfield for the GS mechanism is sequestered from the SUSY breaking by the inflaton sector, so that the soft scalar masses during inflation are not dominated by the $\mathrm{U}(1)_{A} D$-term contribution, it is possible that

$$
\left\langle\phi\left(t_{I}\right)\right\rangle=0,
$$

so the PQ symmetry is restored during inflation, while again

$$
\left\langle\phi\left(t_{0}\right)\right\rangle \sim\left(m_{\mathrm{SUSY}} M_{P l}^{n}\right)^{1 /(n+1)},
$$

in the present universe. In this case, the model is free from the isocurvature and nonGaussianity constraints, however required to have the axion domain-wall number $N_{\mathrm{DW}}=1$, which is a non-trivial constraint on the model building. Furthermore, if one adopts the recent simulation for the axion production by axionic strings and domain walls [60, 61], only the following narrow window of the axion decay constant

$$
10^{9} \mathrm{GeV} \lesssim f_{a}\left(t_{0}\right) \lesssim(\text { a few }) \times 10^{10} \mathrm{GeV}
$$

is allowed by the astrophysical and cosmological constraints, where the relic axions can account for the total dark matter energy density when $f_{a}\left(t_{0}\right)$ saturates the upper bound.

Our results have an intriguing implication for the size of SUSY breaking soft masses in the present universe. Regardless of whether the PQ symmetry is broken or not during inflation, the cosmologically allowed parameter region for a natural axion misalignment angle $\theta_{0}=\mathcal{O}(1)$ points to two possibilities: ${ }^{3}$

i) Axion scale SUSY: $m_{\mathrm{SUSY}} \sim f_{a}\left(t_{0}\right) \sim 10^{9}-10^{11} \mathrm{GeV}$,

ii) Low scale SUSY: $m_{\mathrm{SUSY}} \sim f_{a}^{2}\left(t_{0}\right) / M_{P l} \sim 10^{3}-10^{4} \mathrm{GeV}$.

The results for the case of broken PQ symmetry during inflation suggest also that the axion isocurvature density perturbations have an amplitude close to the present observational bound.

The organization of this paper is as follows. In section 2, we review the relevant features of the string theoretic QCD axion. In section 3, we examine the cosmological constraints on the QCD axion, while taking into account that the axion decay constant during inflation can be much higher than the present value. Although we consider here a specific type of string motivated models, it should be noted that our results apply to generic supersymmetric axion models in which the PQ breaking scale is generated by SUSY breaking effects. In section 4, we present a simple 4-dimensional supergravity (SUGRA) model involving both the inflaton sector and the $\mathrm{U}(1)_{A}$ sector, and examine possible symmetry breaking patterns during inflation.

\section{String theoretic QCD axion}

String theory contains a variety of higher-dimensional antisymmetric $p$-form gauge fields $C_{p}$, together with the associated gauge symmetry, under which

$$
C_{p} \rightarrow C_{p}+d \Lambda_{p-1}
$$

\footnotetext{
${ }^{3}$ The possibility of the axion scale SUSY was noticed also in ref. [62] recently.
} 
where $\Lambda_{p-1}$ is a $(p-1)$-form parameterizing the gauge transformation. For compactifications involving a $p$-cycle $\alpha_{p}$ in the internal space, the resulting 4 -dimensional effective theory contains an axion-like field $\theta_{\text {st }}$ :

$$
C_{p}(x, y)=\theta_{\text {st }}(x) \omega_{p}(y)
$$

where $x$ and $y$ denote the coordinates of the 4-dimensional flat Minkowski spacetime and the internal space, respectively, and $\omega_{p}$ is a harmonic $p$-form with $\int_{\alpha_{p}} \omega_{p}=1$. Since $\omega_{p}(y)=d \Omega_{p-1}(y)$ locally, the shift symmetry

$$
\mathrm{U}(1)_{\text {shift }}: \theta_{\text {st }}(x) \rightarrow \theta_{\text {st }}(x)+\text { constant }
$$

is locally equivalent to the gauge symmetry (2.1), but not globally due to the obstruction from $\int_{\alpha_{p}} \omega_{p} \neq 0$. This implies that the shift symmetry (2.3) is valid in perturbation theory, but can be broken by non-perturbative effects associated with $\int_{\alpha_{p}} \omega_{p} \neq 1$. Such effects include for instance the stringy-instantons wrapping $\alpha_{p}$, as well as the axion couplings to the low energy gauge field instantons, which are induced as

$$
\int C_{p} \wedge F \wedge F \rightarrow \int_{M_{4}} \theta_{\mathrm{st}} F \wedge F \int_{\alpha_{p}} \omega_{p}
$$

It is then a conceivable possibility that stringy instanton effects are negligible for the shift symmetry (2.3), at least in the limit that the $p$-cycle $\alpha_{p}$ is large enough. This would allow that the shift symmetry (2.3) is explicitly broken dominantly by the QCD anomaly, and so the stringy axion $\theta_{\text {st }}$ can be identified as the QCD axion solving the strong CP problem.

A characteristic feature of such string theoretic axion is that its decay constant is of the order of $M_{P l} / 8 \pi^{2}$ if the compactification scale is comparable to the Planck scale, where $8 \pi^{2}$ is a conventional factor for the axion decay constant. To see this, one can consider the 4-dimensional effective SUGRA of the modulus-axion superfield

$$
T=\frac{1}{2} \tau+i \theta_{\text {st }}
$$

where $\tau$ is the modulus partner of $\theta_{\text {st }}$, describing the volume of the $p$-cycle $\alpha_{p}$. For the modulus Kähler potential $K_{0}$ and the holomorphic gauge kinetic function $\tilde{f}_{\alpha}$ for the QCD, which generically take the form,

$$
K=K_{0}\left(T+T^{*}\right), \quad \tilde{f}_{\alpha}=T+\cdots,
$$

the effective lagrangian of $\theta_{\text {st }}$ reads

$$
\begin{aligned}
\mathcal{L}_{\text {eff }} & =M_{P l}^{2} \frac{\partial^{2} K_{0}}{\partial \tau^{2}} \partial_{\mu} \theta_{\mathrm{st}} \partial^{\mu} \theta_{\mathrm{st}}+\frac{1}{4} \theta_{\mathrm{st}} G^{\alpha \mu \nu} \tilde{G}_{\mu \nu}^{\alpha}+\cdots \\
& =\frac{1}{2} \partial_{\mu} a_{\mathrm{st}} \partial^{\mu} a_{\mathrm{st}}+\frac{1}{32 \pi^{2}} \frac{a_{\mathrm{st}}}{f_{a}} G^{a \mu \nu} \tilde{G}_{\mu \nu}^{a}+\cdots,
\end{aligned}
$$

where $a_{\mathrm{st}}$ is the canonically normalized string theoretic QCD axion, $G_{\mu \nu}^{\alpha}$ is the gluon field strength, and the axion decay constant is given by

$$
f_{a}=\frac{1}{8 \pi^{2}}\left(2 \frac{\partial^{2} K_{0}}{\partial \tau^{2}}\right)^{1 / 2} M_{P l}
$$


The BICEP2 results imply that the inflation energy scale is about $10^{16} \mathrm{GeV}$, and therefore the compactification scale is higher than $10^{16} \mathrm{GeV}$. Such a high compactification scale implies that the modulus Kähler metric $\partial^{2} K_{0} / \partial \tau^{2}$ is not significantly smaller than the unity. More specifically, from the QCD gauge kinetic function which depends on $T$, and thereby suggests $\tau \sim 1 / g_{\text {GUT }}^{2}$, the modulus Kähler metric can be estimated as

$$
\left(\frac{\partial^{2} K_{0}}{\partial \tau^{2}}\right)^{1 / 2}=\mathcal{O}\left(g_{\mathrm{GUT}}^{2}\right)
$$

This gives

$$
f_{a}=\mathcal{O}\left(g_{\mathrm{GUT}}^{2} M_{P l} / 8 \pi^{2}\right),
$$

which turns out to be a correct estimate for the most of compactification models ${ }^{4}$ with a compactification scale higher than $10^{16} \mathrm{GeV}$. It has been known for many years that this type of string theoretic QCD axion is subject to severe cosmological constraints. As we will see in section 3, it appears to be ruled out now by the detection of tensor modes by BICEP2 and the PLANCK constraints on isocurvature density perturbations.

In fact, the QCD axion can have a decay constant far below $M_{P l} / 16 \pi^{2}$ even when the compactification scale is comparable to the Planck scale. An attractive scheme to realize such possibility is that the stringy axion $\theta_{\mathrm{st}}$ is charged under an anomalous $\mathrm{U}(1)_{A}$ gauge symmetry, and its modulus partner $\tau$ is stabilized at a value near the point of vanishing FI term. ${ }^{5}$ Indeed, such scheme can be realized in many string compactification models, including the Type II string models with $D$-branes and the heterotic string models with U(1) Yang-Mills bundles on Calabi-Yau manifold. Four-dimensional symmetries of this type of models include a shift symmetry

$$
\mathrm{U}(1)_{\text {shift }}: \quad T \rightarrow T+i c \quad(c=\text { real constant }),
$$

which is broken dominantly by the QCD anomaly, as well as an anomalous $\mathrm{U}(1)_{A}$ gauge symmetry:

$$
\mathrm{U}(1)_{A}: \quad V_{A} \rightarrow V_{A}+\Lambda+\Lambda^{*}, \quad T \rightarrow T+\delta_{\mathrm{GS}} \Lambda, \quad \phi_{i} \rightarrow e^{q_{i} \Lambda} \phi_{i},
$$

where $V_{A}$ is the vector superfield for the $\mathrm{U}(1)_{A}$ gauge multiplet, $\phi_{i}$ are generic $\mathrm{U}(1)_{A^{-}}$ charged chiral matter superfields, $\Lambda$ is a chiral superfield parameterizing $\mathrm{U}(1)_{A}$ transformation on the superspace, and

$$
\delta_{\mathrm{GS}}=\frac{1}{8 \pi^{2}} \sum_{i} q_{i} \operatorname{Tr}\left(T_{a}^{2}\left(\phi_{i}\right)\right)
$$

represents the coefficient of the mixed $\mathrm{U}(1)_{A^{-}} \mathrm{SU}(3)_{c^{-}} \mathrm{SU}(3)_{c}$ anomaly which is cancelled by the GS mechanism.

\footnotetext{
${ }^{4}$ One may be able to obtain a much lower axion scale, while keeping the cutoff-scale for the inflaton sector higher than $10^{16} \mathrm{GeV}$, if the axion sector and the inflaton sector are separated from each other in a warped internal space [63, 64]. Here we do not pursue this kind of more involved possibility.

${ }^{5}$ See refs. $[65,66]$ for string axions with vanishing FI term in the large volume scenario.
} 
Generically the Kähler potential and the QCD gauge kinetic function take the form,

$$
\begin{aligned}
K & =K_{0}\left(T+T^{*}-\delta_{\mathrm{GS}} V_{A}\right)+Z_{i}\left(T+T^{*}-\delta_{\mathrm{GS}} V_{A}\right) \phi_{i}^{*} e^{-q_{i} V_{A}} \phi_{i}+\cdots \\
\tilde{f}_{\alpha} & =T+\cdots
\end{aligned}
$$

In the following, for simplicity, we will consider only a single $\mathrm{U}(1)_{A}$-charged matter field $\phi$ under the assumption that its Kähler metric is a moduli-independent constant. Then the relevant part of the effective lagrangian is given by

$$
\begin{aligned}
\mathcal{L}_{\mathrm{eff}}= & -\frac{1}{4 g_{A}^{2}} F^{\mu \nu} F_{\mu \nu}+M_{P l}^{2} \frac{\partial^{2} K_{0}}{\partial \tau^{2}}\left(\partial_{\mu} \theta_{\mathrm{st}}-\delta_{\mathrm{GS}} A_{\mu}\right)^{2}+D_{\mu} \phi^{*} D^{\mu} \phi \\
& +\frac{1}{2} g_{A}^{2}\left(\delta_{\mathrm{GS}} \frac{\partial K_{0}}{\partial \tau}-|\phi|^{2}\right)^{2}+\frac{1}{4}\left(\theta_{\mathrm{st}}-\delta_{\mathrm{GS}} \arg (\phi)\right) G^{a \mu \nu} \tilde{G}_{\mu \nu}^{a}+\cdots,
\end{aligned}
$$

where we have set $Z_{\phi}=q_{\phi}=1$, and included the counter term for the mixed $\mathrm{U}(1)_{A^{-}}$ $\mathrm{SU}(3)_{c}-\mathrm{SU}(3)_{c}$ anomaly, whose $\mathrm{U}(1)_{A}$ variation is cancelled by the gauge variation of $\theta_{\mathrm{st}}$. The above effective lagrangian can be rewritten as

$$
\begin{aligned}
\mathcal{L}_{\mathrm{eff}}= & -\frac{1}{4 g_{A}^{2}} F^{\mu \nu} F_{\mu \nu}+\frac{1}{2}\left(\left(8 \pi^{2} \delta_{\mathrm{GS}} f_{\mathrm{st}}\right)^{2}+v^{2}\right)\left(\frac{\partial_{\mu} \chi}{\sqrt{\left(8 \pi^{2} \delta_{\mathrm{GS}} f_{\mathrm{st}}\right)^{2}+v^{2}}}-A_{\mu}\right)^{2} \\
& +\frac{1}{2}\left(\partial_{\mu} a\right)^{2}+\frac{1}{32 \pi^{2}} \frac{a}{f_{a}} G \tilde{G}+\frac{1}{2} g_{A}^{2}\left(\xi_{\mathrm{FI}}-v^{2} / 2\right)^{2}+\cdots
\end{aligned}
$$

for $\theta_{\phi}=\arg (\phi)$, and $v=\sqrt{2}\langle\phi\rangle$. Here $\chi$ and $a$ are given by

$$
\begin{aligned}
& \chi=\frac{1}{\sqrt{\left(8 \pi^{2} \delta_{\mathrm{GS}} f_{\mathrm{st}}\right)^{2}+v^{2}}}\left(\left(8 \pi^{2} \delta_{\mathrm{GS}} f_{\mathrm{st}}\right)^{2} \frac{\theta_{\mathrm{st}}}{\delta_{\mathrm{GS}}}+v^{2} \theta_{\phi}\right), \\
& a=\frac{\left(8 \pi^{2} \delta_{\mathrm{GS}} f_{\mathrm{st}}\right) v}{\sqrt{\left(8 \pi^{2} \delta_{\mathrm{GS}} f_{\mathrm{st}}\right)^{2}+v^{2}}}\left(\frac{\theta_{\mathrm{st}}}{\delta_{\mathrm{GS}}}-\theta_{\phi}\right),
\end{aligned}
$$

with $f_{\text {st }}$ and $f_{a}$ defined by

$$
\begin{aligned}
f_{\mathrm{st}} & \equiv \frac{1}{8 \pi^{2}} \sqrt{2 \frac{\partial^{2} K_{0}}{\partial \tau^{2}}} M_{P l}, \\
f_{a} & \equiv \frac{f_{\mathrm{st}} v}{\sqrt{\left(8 \pi^{2} \delta_{\mathrm{GS}} f_{\mathrm{st}}\right)^{2}+v^{2}}} .
\end{aligned}
$$

Note that the $\mathrm{U}(1)_{A} D$-term includes the moduli-dependent FI term,

$$
\xi_{\mathrm{FI}}=\delta_{\mathrm{GS}} \frac{\partial K_{0}}{\partial \tau} M_{P l}^{2} .
$$

Obviously $\chi$ corresponds to the longitudinal component of the massive $\mathrm{U}(1)_{A}$ gauge boson with a mass

$$
M_{A}=g_{A} \sqrt{\left(8 \pi^{2} \delta_{\mathrm{GS}} f_{\mathrm{st}}\right)^{2}+v^{2}},
$$

while $a$ is the physical QCD axion and $f_{a}$ is its decay constant. When the compactification scale is higher than $10^{16} \mathrm{GeV}$, the modulus Kähler metric typically has a vacuum value as 
$\left\langle\partial^{2} K_{0} / \partial \tau^{2}\right\rangle \sim 1 /\langle\tau\rangle^{2}$, and the gauge coupling constant is given by $1 / g_{\text {GUT }}^{2}=\langle\tau\rangle / 2+\cdots$. Thus $f_{\mathrm{st}}$ is around $g_{\mathrm{GUT}}^{2} M_{\mathrm{Pl}} / 8 \pi^{2}$, or it may be possible to increase it by one order of magnitude [24], implying

$$
f_{\mathrm{st}}=\mathcal{O}\left(10^{-1}-10^{-2}\right) \times M_{P l},
$$

independently of the details of moduli stabilization.

On the other hand, the matter vacuum expectation value $v=\sqrt{2}\langle\phi\rangle$ severely depends on the mechanism of moduli stabilization, particularly on the vacuum value of the FI term. In 4-dimensional $N=1$ SUGRA with $m_{3 / 2} \ll M_{A}$ for $m_{3 / 2}$ being the gravitino mass, we have the following bound on the $D$-term:

$$
\left|D_{A}\right|=\left|\xi_{\mathrm{FI}}-v^{2} / 2\right| \lesssim \mathcal{O}\left(\frac{m_{3 / 2}^{2} M_{P l}^{2}}{M_{A}^{2}}\right),
$$

which can be derived from the stationary condition for the scalar potential [67,68]. Then there are essentially two distinctive possibilities. One is that the modulus $\tau$ is stabilized at a value with

$$
\frac{\partial K_{0}}{\partial \tau}=\mathcal{O}(1)
$$

which is the case, for instance, when $\theta_{\text {st }}$ is the model-independent axion and $\tau$ is the dilaton in the heterotic string theory. In this case, we have

$$
\xi_{\mathrm{FI}} \simeq v^{2}=\mathcal{O}\left(\delta_{\mathrm{GS}} M_{P l}^{2}\right)>f_{\mathrm{st}}^{2}=\mathcal{O}\left(M_{P l}^{2} /\left(8 \pi^{2}\right)^{2}\right) .
$$

Then the physical QCD axion is mostly $\theta_{\text {st }}$ which originates from antisymmetric tensor gauge fields, and its decay constant reads

$$
f_{a}=\frac{f_{\mathrm{st}} v}{\sqrt{\left(8 \pi^{2} \delta_{\mathrm{GS}} f_{\mathrm{st}}\right)^{2}+v^{2}}} \simeq f_{\mathrm{st}} .
$$

Axion cosmology in this case is essentially the same as in the case without anomalous $\mathrm{U}(1)_{A}$ symmetry, and therefore the model is in conflict with the inflation scale $H_{I} \simeq 10^{14} \mathrm{GeV}$.

Another, more interesting, possibility is that the modulus $\tau$ is stabilized at a value near the point of vanishing FI-term. Most of the known models with anomalous $\mathrm{U}(1)_{A}$ symmetry, realized either in the Type II string theory with $D$-branes or in the heterotic string theory with $\mathrm{U}(1)$ gauge bundles, admit a supersymmetric solution with

$$
\xi_{\mathrm{FI}}=\phi=0 .
$$

To be phenomenologically viable, this solution should be destabilized by a tachyonic SUSY breaking mass of $\phi$ to develop $v>10^{9} \mathrm{GeV}$. Schematically the scalar potential of $\phi$ takes the form

$$
V(\phi)=-m_{\mathrm{SUSY}}^{2}|\phi|^{2}+\left|\frac{\partial W}{\partial \phi}\right|^{2}=-m_{\mathrm{SUSY}}^{2}|\phi|^{2}+\frac{1}{M_{P l}^{2 n}}|\phi|^{4+2 n} \quad(n \geq 0),
$$

yielding

$$
v \sim\left(m_{\mathrm{SUSY}} M_{P l}^{n}\right)^{1 /(n+1)} \ll f_{\mathrm{st}},
$$


where the SUSY breaking mass $m_{\mathrm{SUSY}}$ is assumed to be small enough compared to $M_{P l}$. In this case, the physical QCD axion is mostly $\theta_{\phi}=\arg (\phi)$, and the axion decay constant is determined by $v$,

$$
f_{a}=\frac{f_{\mathrm{st}} v}{\sqrt{\left(8 \pi^{2} \delta_{\mathrm{GS}} f_{\mathrm{st}}\right)^{2}+v^{2}}} \simeq \frac{v}{8 \pi^{2} \delta_{\mathrm{GS}}},
$$

where $8 \pi^{2} \delta_{\mathrm{GS}}=\sum_{i} q_{i} \operatorname{Tr}\left(T_{a}^{2}\left(\phi_{i}\right)\right)$, and we have set $q_{\phi}=1$.

So far, we have discussed the axion decay constant in the present universe in models with anomalous $\mathrm{U}(1)_{A}$ gauge symmetry. An interesting feature of the axion models discussed above, providing an intriguing connection between the axion scale and SUSY breaking scale:

$$
f_{a}\left(t_{0}\right) \sim v\left(t_{0}\right) \sim\left(m_{\mathrm{SUSY}} M_{P l}^{n}\right)^{1 /(n+1)},
$$

is that the axion decay constant $f_{a}\left(t_{I}\right)$ during inflation can be very different from the present axion decay constant $f_{a}\left(t_{0}\right)$. In regard to this, we have again two distinctive possibilities, which will be discussed in more detail in section 4 :

a) PQ symmetry restored during inflation with $v\left(t_{I}\right)=0$,

b) $\mathrm{PQ}$ symmetry broken at a higher scale with $v\left(t_{I}\right) \sim\left(4 \pi H_{I} M_{P l}^{n}\right)^{1 /(n+1)}$.

In section 3, we will discuss the cosmological constraints on the string theoretic QCD axion charged under an anomalous $\mathrm{U}(1)_{A}$ gauge symmetry, while taking into account this variation of the axion decay constant from the inflationary epoch to the present universe. In section 4, we examine the symmetry breaking pattern during inflation in the context of simple SUGRA model involving both a chaotic inflaton sector and the $\mathrm{U}(1)_{A}$ sector for the QCD axion.

\section{Cosmological constraints}

The QCD axion is subject to various cosmological constraints depending on whether the PQ symmetry is restored or not in the early universe. Let us start with the case where the $\mathrm{PQ}$ symmetry is restored during inflation:

$$
v\left(t_{I}\right)=0
$$

In this case, the domain-wall number $N_{\text {DW }}$ should be equal to one since otherwise domain walls formed during the QCD phase transition will overclose the universe. Even for $N_{\mathrm{DW}}=$ 1 , axionic strings are formed during the $\mathrm{PQ}$ phase transition, and develop into a network of strings attached by domain walls during the QCD phase transition. Then dark matter axions are produced from the annihilations of these topological defects, as well as from the coherent oscillation of misaligned axion field. Putting these together, one finds that the relic axion mass density at present is given by

$$
\begin{aligned}
\Omega_{a} h^{2} & =\left(\Omega_{\text {mis }}+\Omega_{\text {string }}+\Omega_{\text {wall }}\right) h^{2} \\
& \simeq(0.58+(2.0 \pm 1.0)+(5.8 \pm 2.8)) \times\left(\frac{\Lambda_{\mathrm{QCD}}}{400 \mathrm{MeV}}\right)\left(\frac{f_{a}\left(t_{0}\right)}{10^{12} \mathrm{GeV}}\right)^{1.19}
\end{aligned}
$$


where we have used the results of the recent numerical simulation for the axion production from the collapsing string and wall system $[60,61],{ }^{6}$ together with the root-mean-square value of the axion misalignment angle $\left\langle\theta_{0}^{2}\right\rangle \simeq 1.85 \times \pi^{2} / 3$, which takes into account the anharmonic factor 1.85. Combined with astrophysical constraints, the condition $\Omega_{a} \leq \Omega_{\mathrm{DM}}$ determines the allowed range of the axion decay constant as

$$
10^{9} \mathrm{GeV}<f_{a}\left(t_{0}\right)<(2-4) \times 10^{10} \mathrm{GeV},
$$

when the PQ symmetry was restored during inflation, where $\Omega_{\mathrm{DM}} \simeq 0.25$ denotes the total dark matter energy density. Applying this to the previously discussed scheme generating the axion scale as

$$
f_{a}\left(t_{0}\right) \sim\left(m_{\mathrm{SUSY}} M_{P l}^{n}\right)^{1 /(n+1)},
$$

we are led to either the axion scale SUSY $(n=0)$ or the TeV scale SUSY $(n=1)$,

$$
\begin{aligned}
& n=0: m_{\mathrm{SUSY}} \sim 10^{9}-10^{10} \mathrm{GeV}, \\
& n=1: m_{\mathrm{SUSY}} \sim 10^{3} \mathrm{GeV} .
\end{aligned}
$$

Another, presumably more interesting, scenario is that the PQ symmetry is broken during inflation at a scale much higher than the present axion scale. In such case, there are no topological defects, but the axion can still cause cosmological problems since during inflation it acquires quantum fluctuations

$$
\delta a\left(t_{I}\right)=\frac{H_{I}}{2 \pi}
$$

for the canonically normalized axion field during inflation, $a\left(t_{I}\right)=f_{a}\left(t_{I}\right) \theta_{a}$. In models with anomalous $\mathrm{U}(1)_{A}$ gauge symmetry, one combination of the GS axion $\theta_{\mathrm{st}}$ and the matter field axion $\theta_{\phi}=\arg (\phi)$ becomes the longitudinal component of the massive $\mathrm{U}(1)_{A}$ gauge boson having a mass much larger than $H_{I} \simeq 10^{14} \mathrm{GeV}$, while the other $\mathrm{U}(1)_{A}$-invariant combination can be identified as the QCD axion. The fraction of each component in the QCD axion changes with time, and the main component during and after inflation are different if $v\left(t_{0}\right) \ll f_{\text {st }} \lesssim v\left(t_{I}\right)$. The axion fluctuation around the average misalignment at the moment of coherent oscillation is given by

$$
\left\langle\delta \theta^{2}\right\rangle=\left\langle\frac{\delta a^{2}\left(t_{0}\right)}{f_{a}^{2}\left(t_{0}\right)}\right\rangle=\left(\frac{H_{I}}{2 \pi f_{a}\left(t_{I}\right)}\right)^{2}
$$

where $\delta \theta=\delta a\left(t_{0}\right) / f_{a}\left(t_{0}\right)=\delta a\left(t_{I}\right) / f_{a}\left(t_{I}\right)$ has been used. For axion models with $\mathrm{U}(1)_{A}$, the ratio between the axion scales during and after inflation is estimated to be

$$
\frac{f_{a}\left(t_{I}\right)}{f_{a}\left(t_{0}\right)} \simeq \frac{v\left(t_{I}\right)}{v\left(t_{0}\right)}\left(\frac{\left(8 \pi^{2} \delta_{\mathrm{GS}} f_{\mathrm{st}}\right)^{2}+v^{2}\left(t_{0}\right)}{\left(8 \pi^{2} \delta_{\mathrm{GS}} f_{\mathrm{st}}\right)^{2}+v^{2}\left(t_{I}\right)}\right)^{1 / 2},
$$

\footnotetext{
${ }^{6}$ Axion radiation by the string-wall system is determined mostly by the string and wall tensions given by $\mu_{s} \sim f_{a}^{2} \ln \left(m_{\phi} t\right)$ and $\sigma_{w} \sim m_{a} f_{a}^{2}$, where $m_{\phi}$ is the mass of the PQ breaking field. It was assumed that $m_{\phi} \sim f_{a}$ in refs. [60,61], while in our case $m_{\phi} \sim m_{\mathrm{SUSY}}$. This may cause a non-negligible change of the axion mass density produced by the string-wall system. As it does not change the order of magnitude of the axion mass density, we ignore this point in the present discussion.
} 
where we have used the relations (2.18) and (2.19). Note that the expectation value of $f_{\mathrm{st}}=\sqrt{2 \partial_{\tau}^{2} K_{0}} M_{P l} / 8 \pi^{2}$ does not change significantly during and after inflation as the GS modulus $\tau$ is stabilized by the $\mathrm{U}(1)_{A} D$-term potential at a value near the point of vanishing FI term in both periods, with a superheavy mass $M_{\tau} \sim \delta_{\mathrm{GS}} M_{P l}$. It is also important to note that $f_{a}\left(t_{I}\right) / f_{a}\left(t_{0}\right)$ is always smaller than about $f_{\text {st }} / v\left(t_{0}\right)$ for $v\left(t_{0}\right) \ll v\left(t_{I}\right)$.

The axion field is uniform, $a\left(t_{0}\right)=f_{a}\left(t_{0}\right) \theta_{0}$, at the classical level throughout the whole observable universe if the PQ symmetry were broken during inflation. In addition to this misalignment, there are axion fluctuations $\delta a\left(t_{0}\right)$ induced during inflation, which are subject to various cosmological constraints. Let us summarize the constraints, which depend on the values of $\theta_{0}, f_{a}\left(t_{0}\right), f_{a}\left(t_{I}\right), H_{I}$, and $\Omega_{a} / \Omega_{\mathrm{DM}}$. We first have the obvious condition:

$$
\frac{\Omega_{a}}{\Omega_{\mathrm{DM}}} \simeq 0.11\left(\theta_{0}^{2}+\left\langle\delta \theta^{2}\right\rangle\right)\left(\frac{\Lambda_{\mathrm{QCD}}}{400 \mathrm{MeV}}\right)\left(\frac{f_{a}\left(t_{0}\right)}{10^{11} \mathrm{GeV}}\right)^{1.19} \leq 1,
$$

neglecting anharmonic effects, which become important if the axion initial position is very close to the hilltop of the potential. Note that one cannot avoid the contribution from the axion fluctuation $\left\langle\delta \theta^{2}\right\rangle \propto H_{I}^{2}$. The QCD axion obtains mass after the QCD phase transition. Then its fluctuations lead to isocurvature density perturbations of axion dark matter and also to non-Gaussianity [30-34], which are strongly constrained by the observed CMB power spectrum.

The power spectrum of axion isocurvature perturbations is given by [35-37]

$$
\begin{aligned}
\mathcal{P}_{S} & =2\left(\frac{\Omega_{a}}{\Omega_{\mathrm{DM}}}\right)^{2} \frac{2 \theta_{0}^{2}+\left\langle\delta \theta^{2}\right\rangle}{\left(\theta_{0}^{2}+\left\langle\delta \theta^{2}\right\rangle\right)^{2}}\left\langle\delta \theta^{2}\right\rangle \\
& \simeq \frac{0.44}{x}\left(\frac{\Omega_{a}}{\Omega_{\mathrm{DM}}}\right)\left(\frac{H_{I}}{2 \pi f_{a}\left(t_{I}\right)}\right)^{2}\left(\frac{f_{a}\left(t_{0}\right)}{10^{11} \mathrm{GeV}}\right)^{1.19}
\end{aligned}
$$

where we have used the relation (3.8) with $\Lambda_{\mathrm{QCD}} \simeq 400 \mathrm{MeV}$, and $x$ is defined by

$$
x \equiv 2 \frac{\theta_{0}^{2}+\left\langle\delta \theta^{2}\right\rangle}{2 \theta_{0}^{2}+\left\langle\delta \theta^{2}\right\rangle}=1-2 .
$$

The isocurvature power is constrained by the Planck observations [57] to be

$$
\frac{\mathcal{P}_{S}}{\mathcal{P}_{\zeta}}<0.041 \text { (95\% C.L.) }
$$

where $\mathcal{P}_{\zeta} \simeq 2.19 \times 10^{-9}$ is the power spectrum of the curvature perturbations. Then the isocurvature constraint reads

$$
\left(\frac{\Omega_{a}}{\Omega_{\mathrm{DM}}}\right)\left(\frac{f_{a}\left(t_{0}\right)}{10^{11} \mathrm{GeV}}\right)^{1.19}<2 \times 10^{-10} x\left(\frac{H_{I}}{2 \pi f_{a}\left(t_{I}\right)}\right)^{-2} .
$$

In addition, there appears non-Gaussianity in isocurvature fluctuations [74], and the experimental bound is roughly translated into

$$
\left(\frac{\Omega_{a}}{\Omega_{\mathrm{DM}}}\right)^{1 / 2}\left(\frac{f_{a}\left(t_{0}\right)}{10^{11} \mathrm{GeV}}\right)^{1.19} \lesssim 10^{-6}\left(\frac{H_{I}}{2 \pi f_{a}\left(t_{I}\right)}\right)^{-2} .
$$


Finally, the existence of the average misalignment angle $\theta_{0}$ contributing to the relic axion abundance leads to the condition

$$
\frac{\Omega_{a}}{\Omega_{\mathrm{DM}}} \gtrsim 0.11\left(\frac{H_{I}}{2 \pi f_{a}\left(t_{I}\right)}\right)^{2}\left(\frac{f_{a}\left(t_{0}\right)}{10^{11} \mathrm{GeV}}\right)^{1.19},
$$

taking $\Lambda_{\mathrm{QCD}} \simeq 400 \mathrm{MeV}$. When combined with this, the isocurvature constraint (3.12) puts a severe upper bound on the axion decay constant at present:

$$
f_{a}\left(t_{0}\right)<7.1 \times 10^{13} \mathrm{GeV}\left(\frac{H_{I}}{10^{14} \mathrm{GeV}}\right)^{-1.68}\left(\frac{f_{a}\left(t_{I}\right)}{10^{17} \mathrm{GeV}}\right)^{1.68},
$$

which applies independently of the value of $\Omega_{a}$. Similarly, the constraint (3.13) from nonGaussianity can be combined with (3.14) to give a upper bound on $f_{a}\left(t_{0}\right)$, however the resulting bound is always weaker than the bound (3.15) from isocurvature perturbations. This implies that, it is the isocurvature constraint that determines the cosmologically viable range of the axion mass density and decay constant.

Before going further, let us discuss anharmonic effects, which have been neglected so far. The axion abundance produced from the coherent oscillation is enhanced if the initial axion position is close to the hilltop [69-73], where the axion potential is not approximated by a quadratic potential. Such effects can be included by taking

$$
\left\langle\theta^{2}\right\rangle \rightarrow\left\langle F\left(\theta^{2}\right) \theta^{2}\right\rangle
$$

in the relation for the axion density (3.8), with $F$ given by [72]

$$
F(z) \simeq\left(\ln \left(\frac{e}{1-z / \pi^{2}}\right)\right)^{1.19}
$$

for $0 \leq z<\pi^{2}$. The anharmonicity factor $F(z)$ increases from unity as $z$ increases. The axion contribution to isocurvature density perturbations is also enhanced as the initial position approaches the hilltop. One can estimate it using that the axion abundance is proportional to $F\left(\theta^{2}\right) \theta^{2}[73]$,

$$
\mathcal{P}_{S}=\left(1+\frac{F^{\prime}\left(\theta_{0}^{2}\right)}{F\left(\theta_{0}^{2}\right)}\right)^{2} \times 4\left(\frac{\Omega_{a}}{\Omega_{\mathrm{DM}}}\right)^{2} \frac{\left\langle\delta \theta^{2}\right\rangle}{\theta_{0}^{2}}\left(1+\mathcal{O}\left(\frac{\left\langle\delta \theta^{2}\right\rangle}{\theta_{0}^{2}}\right)\right),
$$

where $F^{\prime} \equiv z d F(z) / d z$. Thus the isocurvature perturbation is enhanced approximately by the factor, $\left(1+F^{\prime} / F\right)^{2}$, for small fluctuations $\left\langle\delta \theta^{2}\right\rangle \ll \theta_{0}^{2}$. Including this enhancement factor, one finds that the upper bound on $\Omega_{a}$ from the isocurvature constraint is smaller than the value obtained by the relation (3.12).

Obviously high scale inflation puts strong constraints on the possible range of the axion scale and relic abundance, which may be satisfied by having a larger decay constant during inflation [75]. As will be discussed in more detail in section 4, in models with anomalous $\mathrm{U}(1)_{A}$ gauge symmetry, one can easily obtain

$$
f_{a}\left(t_{I}\right) \sim f_{\mathrm{st}}=\frac{1}{8 \pi^{2}} \sqrt{2 \frac{\partial^{2} K_{0}}{\partial \tau^{2}}} M_{P l}=\mathcal{O}\left(10^{-1}-10^{-2}\right) \times M_{P l},
$$




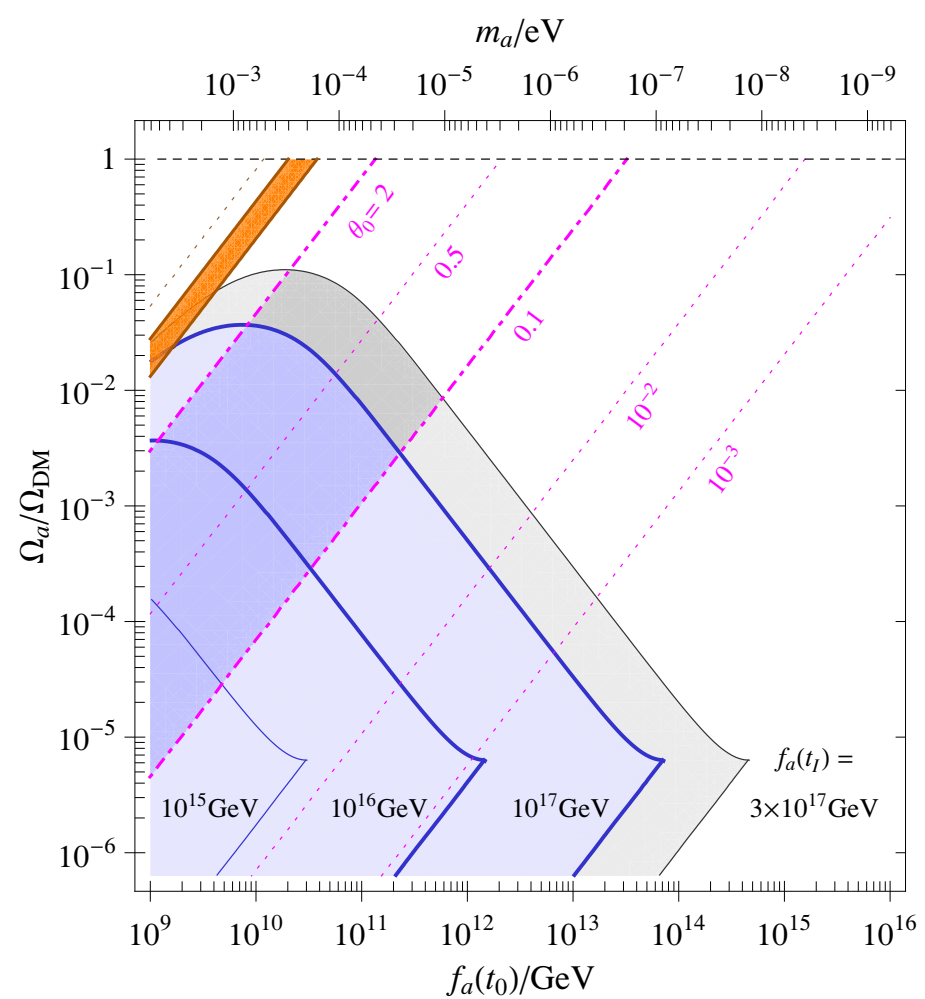

Figure 1. The axion decay constant (or, equivalently, the axion mass) and the axion fraction of the dark matter energy density in the present universe, which are consistent with cosmological constraints. The orange band is the allowed region when the PQ symmetry is restored during inflation or reheating, for which the domain-wall number should be equal to one. For the case that the PQ symmetry were broken during and after inflation, there are no topological defects, but the axion abundance and decay constant are severely constrained by the bound on isocurvature density perturbations. These cosmological constraints are relaxed in models with anomalous $\mathrm{U}(1)_{A}$ gauge symmetry since the axion decay constant during inflation can be much higher than the present value. For the inflation scale $H_{I}=10^{14} \mathrm{GeV}$, the shaded region bounded by the solid lines for $f_{a}\left(t_{I}\right)=10^{15}, 10^{16}, 10^{17}, 3 \times 10^{17} \mathrm{GeV}$ are cosmologically allowed. We also show the average axion misalignment angle $\theta_{0}$ in dot-dashed and dotted lines.

with

$$
v\left(t_{I}\right) \sim\left(\sqrt{8 \pi^{2}} H_{I} M_{P l}^{n}\right)^{1 /(n+1)} \gtrsim f_{\mathrm{st}},
$$

for a reasonable range of model parameters and $H_{I} \sim 10^{14} \mathrm{GeV}$. Then the axion fluctuation (3.6) is suppressed by a factor $v\left(t_{0}\right) / f_{\text {st }}$ insensitively to the precise value of $v\left(t_{I}\right)$, relaxing the associated cosmological constraints.

Figure 1 shows the region consistent with the cosmological constraints in the plane of $f_{a}\left(t_{0}\right)$ (or, equivalently, the axion mass $m_{a}$ ) and $\Omega_{a} / \Omega_{\mathrm{DM}}$, for $H_{I}=10^{14} \mathrm{GeV}$. The orange band, which is obtained from the relation (3.2), shows the allowed range for the case that the PQ symmetry is restored during inflation or reheating. In such case, the axion domain wall number should be equal to one. On the other hand, if the PQ symmetry remains broken during and after inflation, the model is free from topological defects, but 
the axion fluctuations produced during inflation put strong constraints. The left side of the solid curves represents the allowed region for $f_{a}\left(t_{I}\right)=10^{15}, 10^{16}, 10^{17}, 3 \times 10^{17} \mathrm{GeV}$, from left to right, respectively. The allowed region becomes larger as $f_{a}\left(t_{I}\right)$ increases, but the axion cannot account for the total abundance of dark matter for $f_{a}\left(t_{I}\right)$ smaller than the Planck scale [59]. In models with anomalous $\mathrm{U}(1)_{A}$, we have $f_{a}\left(t_{I}\right) \sim f_{\text {st }}=\mathcal{O}\left(10^{16}\right.$ $\left.10^{17}\right) \mathrm{GeV}$ for $v\left(t_{I}\right) \gtrsim f_{\mathrm{st}}$, for which the axion fluctuation is suppressed by $f_{a}\left(t_{0}\right) / f_{a}\left(t_{I}\right) \sim$ $\left(m_{\mathrm{SUSY}} M_{P l}^{n}\right)^{1 /(n+1)} / f_{\mathrm{st}}$. We also show the result for a fixed value of the misalignment angle, $\theta_{0}=2,0.5,0.1,10^{-2}, 10^{-3}$ in the magenta dot-dashed and dotted lines for $f_{a}\left(t_{I}\right)=$ $10^{16} \mathrm{GeV}$. If one takes a larger value of $f_{a}\left(t_{I}\right)$, the dot-dashed lines move to the right, but only slightly for $\theta_{0}^{2} \gg\left(H_{I} / 2 \pi f_{a}\left(t_{I}\right)\right)^{2}$. Here we have included the full anharmonic effects by taking the initial condition, $\left|1-\theta_{0} / \pi\right| \lesssim H_{I} / f_{a}\left(t_{I}\right)$, so that the axion does not pass over the hilltop of the potential in the presence of fluctuations.

We close this section by summarizing the cosmologically viable values of the axion decay constant and relic abundance. The value of $f_{\mathrm{st}}=\sqrt{2 \partial_{\tau}^{2} K_{0}} M_{P l} / 8 \pi^{2}$ lies in the range between about $10^{16}$ and $10^{17} \mathrm{GeV}$, independently of the details of moduli stabilization. Then the isocurvature constraint requires the axion decay constant at present to be

$$
f_{a}\left(t_{0}\right)<7.1 \times 10^{13} \mathrm{GeV}\left(\frac{f_{\mathrm{st}}}{10^{17} \mathrm{GeV}}\right)^{1.68},
$$

as can be seen from the relation (3.15) by taking $f_{a}\left(t_{I}\right) \simeq f_{\text {st }}$, and also from figure 1 , where $f_{a}\left(t_{0}\right)$ around $10^{14} \mathrm{GeV}$ would require a severe fine-tuning of the axion misalignment angle $\theta_{0}$. On the other hand, the natural value of $\theta_{0}$ would be of the order of unity, which corresponds to the dark shaded region between the two dot-dashed lines. For such natural value of $\theta_{0}$, the isocurvature constraint is translated into

$$
f_{a}\left(t_{0}\right)<6 \times 10^{10} \mathrm{GeV}\left(\frac{\theta_{0}}{0.5}\right)^{-0.84}\left(\frac{f_{\mathrm{st}}}{10^{17} \mathrm{GeV}}\right)^{0.84}
$$

Hence, in the case that the PQ symmetry is broken during inflation with a misalignment angle $\theta_{0}=\mathcal{O}(1)$, the QCD axion is expected to have a decay constant in the range around $10^{9}-10^{11} \mathrm{GeV}$, while composing up to $0.1-10 \%$ of the total dark matter energy density. If this were the case, one is again led to either the axion scale SUSY $(n=0)$,

$$
m_{\mathrm{SUSY}} \sim v\left(t_{0}\right) \sim 10^{9}-10^{11} \mathrm{GeV}
$$

or the low scale $\operatorname{SUSY}(n=1)$ with

$$
m_{\mathrm{SUSY}} \sim \frac{v^{2}\left(t_{0}\right)}{M_{P l}} \sim 10^{3}-10^{4} \mathrm{GeV}
$$

because the axion scale is determined by $v\left(t_{0}\right) \sim\left(m_{\mathrm{SUSY}} M_{P l}^{n}\right)^{1 /(n+1)}$, and sizable suppression of axion fluctuations is achieved for $v\left(t_{I}\right) \sim f_{\text {st }}$ (see also section 4). Although unnatural, $\theta_{0}$ may have a value much smaller than one, which would allow a larger axion decay constant as (3.21). In this case, the QCD axions constitute only a negligibly small fraction of the observed dark matter energy density. 


\section{Axion decay constant during and after inflation}

In this section, we examine the PQ symmetry breaking both at present and during inflation in the context of simple supergravity model involving the $\mathrm{U}(1)_{A}$ and inflaton sectors. We begin with a configuration with vanishing FI term,

$$
\left.\xi_{\mathrm{FI}} \propto \frac{\partial K_{0}}{\partial \tau}\right|_{\tau=\tau_{0}}=0,
$$

where $T=\tau / 2+i \theta_{\text {st }}$ is the modulus-axion superfield implementing the GS anomaly cancellation mechanism. For simplicity, we consider a minimal $\mathrm{U}(1)_{A}$ sector involving the vector multiplet $V_{A}$, the GS multiplet $T$, and two matter fields $\phi_{i}(i=1,2)$ with opposite sign of $\mathrm{U}(1)_{A}$ charges. Then, the Kähler potential and superpotential of the $\mathrm{U}(1)_{A}$ sector can be expanded around the configuration $T=\tau_{0} / 2$ and $\phi_{i}=0$ as

$$
\begin{aligned}
K & =\frac{M_{P l}^{2} \partial_{\tau}^{2} K_{0}\left(\tau_{0}\right)}{2}\left(\tau-\tau_{0}-\delta_{\mathrm{GS}} V_{A}\right)^{2}+\phi_{1}^{*} e^{-V_{A}} \phi_{1}+\phi_{2}^{*} e^{(n+2) V_{A}} \phi_{2}, \\
W & =\lambda \frac{\phi_{1}^{n+2} \phi_{2}}{M_{P l}^{n}},
\end{aligned}
$$

where we have assumed that the matter Kähler metric are moduli-independent, and the $\mathrm{U}(1)_{A}$ charges of $\phi_{i}$ are chosen as $q_{1}=1$ and $q_{2}=-(n+2)$.

The $D$-flat direction of the $\mathrm{U}(1)_{A}$ sector is lifted by SUSY breaking effects, and eventually determines the PQ breaking scale as

$$
f_{a}=\frac{f_{\mathrm{st}} v}{\sqrt{\left(8 \pi^{2} \delta_{\mathrm{GS}} f_{\mathrm{st}}\right)^{2}+v^{2}}},
$$

where

$$
\begin{aligned}
f_{\mathrm{st}} & =\frac{\sqrt{2 \partial_{\tau}^{2} K_{0}}}{8 \pi^{2}} M_{P l}, \\
v^{2} & =2 \sum_{i} q_{i}^{2}\left\langle\left|\phi_{i}\right|^{2}\right\rangle=\sum_{i} q_{i}^{2} v_{i}^{2},
\end{aligned}
$$

with $v_{i} \equiv \sqrt{2}\left\langle\left|\phi_{i}\right|\right\rangle$. It is thus important to know how the $D$-flat direction couples to the SUSY breaking sector in the model.

As a concrete example, we introduce a Polonyi-like field $Z$ for the SUSY breaking at present, and an additional field $X$ which develops a large SUSY breaking $F$-term during inflation described by the inflaton superfield $\Phi$. For a large field inflation within the supergravity framework, we assume an approximate shift symmetry, $\Phi \rightarrow \Phi+i c$. Then the Kähler potential and superpotential of the SUSY breaking sector are given by

$$
\begin{aligned}
& K_{\mathrm{SB}}=|Z|^{2}-\frac{|Z|^{4}}{\Lambda^{2}}+\frac{1}{2}\left(\Phi+\Phi^{*}\right)^{2}+|X|^{2}, \\
& W_{\mathrm{SB}}=\omega_{0}+M^{2} Z+\mu X \Phi .
\end{aligned}
$$

Following ref. [76], it is assumed that the inflaton sector fields, $\Phi$ and $X$, are odd under a $Z_{2}$ symmetry, and their superpotential coupling preserves $R$-symmetry, but explicitly 
breaks the shift symmetry of $\Phi$. Note that inflation is driven along the $\operatorname{Im}(\Phi)$ direction by the $F$-term potential of $X$. In the present universe, the inflaton sector fields are settled at $X\left(t_{0}\right)=\Phi\left(t_{0}\right)=0$, and SUSY breaking is due to the $F$-term of the Polonyi-like field:

$$
F^{Z} \simeq \sqrt{3} m_{3 / 2} M_{P l}
$$

where $m_{3 / 2}$ is the gravitino mass in the present universe with nearly vanishing cosmological constant. On the other hand, during inflation, SUSY breaking is dominated by

$$
F^{X} \simeq \mu \varphi\left(t_{I}\right) \simeq \sqrt{3} H_{I} M_{P l},
$$

where $\varphi=\operatorname{Im}(\Phi)$ is the inflaton field, which takes a value larger than the Planck scale to implement the inflation.

The potential for the $D$-flat direction is generated from the coupling between the $\mathrm{U}(1)_{A}$ sector and the SUSY breaking sector, which generically take the form,

$$
\Delta K=\left(k|Z|^{2}+\kappa|X|^{2}\right)\left(\tau-\tau_{0}-\delta_{\mathrm{GS}} V_{A}\right)+\frac{k_{i}|Z|^{2}+\kappa_{i}|X|^{2}}{M_{P l}^{2}} \phi_{i}^{*} e^{-q_{i} V_{A}} \phi_{i},
$$

when expanded around $T=\tau_{0} / 2$ and $\phi_{i}=0$. After integrating out the $F$-term SUSY breaking by $F^{Z, X}$, the scalar potential relevant to the stabilization of the $D$-flat direction is given by

$$
\begin{aligned}
V \simeq & \frac{g_{A}^{2}}{2} D_{A}^{2}+V_{0}(\tau)+(n+2)^{2} \lambda^{2} \frac{\left|\phi_{1}\right|^{2(n+1)}\left|\phi_{2}\right|^{2}}{M_{P l}^{2 n}}+\lambda^{2} \frac{\left|\phi_{1}\right|^{2(n+2)}}{M_{P l}^{2 n}} \\
& +m_{1}^{2}\left|\phi_{1}\right|^{2}+m_{2}^{2}\left|\phi_{2}\right|^{2}-\left(\lambda A_{\phi} \frac{\phi_{1}^{n+2} \phi_{2}}{M_{P l}^{n}}+\text { h.c. }\right),
\end{aligned}
$$

with

$$
\begin{aligned}
D_{A} & =\left|\phi_{1}\right|^{2}-(n+2)\left|\phi_{2}\right|^{2}-\delta_{\mathrm{GS}} \frac{\partial K_{0}}{\partial \tau}, \\
V_{0} & =e^{K_{0}}\left(\frac{M^{4}}{1+k\left(\tau-\tau_{0}\right)}+\frac{\mu^{2} \varphi^{2}}{1+\kappa\left(\tau-\tau_{0}\right)}-3\left|W_{\mathrm{SB}}\right|^{2}\right),
\end{aligned}
$$

where $m_{i}^{2}$ parameterize the soft scalar masses generated by the $F$-term SUSY breaking. It is clear that the phase of $\phi_{1}^{n+2} \phi_{2}$ is fixed by the $A$-term alone. Using this, one can always take a field basis such that $A_{\phi}$ is real and positive. From the above scalar potential, we find the stationary conditions to be

$$
\begin{aligned}
& \partial_{\tau} V \propto g_{A}^{2} D_{A}-\frac{1}{\delta_{\mathrm{GS}} \partial_{\tau}^{2} K_{0}}\left(\frac{\partial V_{0}}{\partial \tau}+\cdots\right)=0, \\
& \partial_{\phi_{1}} V \propto\left|\phi_{1}\right|\left(G_{1}\left(\left|\phi_{i}\right|, \tau\right)-(n+2) \lambda A_{\phi} \frac{\left|\phi_{1}\right|^{n}\left|\phi_{2}\right|}{M_{P l}^{n}}\right)=0, \\
& \partial_{\phi_{2}} V \propto\left|\phi_{2}\right| G_{2}\left(\left|\phi_{i}\right|, \tau\right)-\lambda A_{\phi} \frac{\left|\phi_{1}\right|^{n+2}}{M_{P l}^{n}}=0,
\end{aligned}
$$

for $G_{i}$ given by

$$
G_{1}=g_{A}^{2} D_{A}+m_{1}^{2}+(n+1)(n+2)^{2} \lambda^{2} \frac{\left|\phi_{1}\right|^{2 n}\left|\phi_{2}\right|^{2}}{M_{P l}^{2 n}}+(n+2) \lambda^{2} \frac{\left|\phi_{1}\right|^{2 n+2}}{M_{P l}^{2 n}}+\cdots,
$$




$$
G_{2}=-(n+2) g_{A}^{2} D_{A}+m_{2}^{2}+(n+2)^{2} \lambda^{2} \frac{\left|\phi_{1}\right|^{2 n+2}}{M_{P l}^{2 n}}+\cdots
$$

where the ellipsis indicates terms of higher order in $\left|\phi_{i}\right|^{2}$ and $\left(\tau-\tau_{0}\right)$. Among the three pseudo-scalar fields,

$$
\theta_{\mathrm{st}}=\operatorname{Im}(T), \quad \theta_{1}=\arg \left(\phi_{1}\right), \quad \theta_{2}=\arg \left(\phi_{2}\right),
$$

the combination $\theta_{2}+(n+2) \theta_{1}$ is stabilized by the $A$-term, while the other two remain massless. One of them is absorbed into the $\mathrm{U}(1)_{A}$ gauge boson, and the other corresponds to the QCD axion.

Let us now examine the vacuum configuration in the present universe with $X=\Phi=0$, and the resulting axion decay constant. First, the condition $\partial_{\tau} V=0$ reads

$$
g_{A}^{2} D_{A}-\frac{1}{\delta_{\mathrm{GS}} \partial_{\tau}^{2} K_{0}}\left(\frac{\partial K_{0}}{\partial \tau}-\frac{k}{1+\left(\tau-\tau_{0}\right) k}\right) V_{0}=0
$$

where $k$ is the coupling between the GS modulus-axion multiplet $T=\tau / 2+i \theta_{\text {st }}$ and the Polony-like field $Z$ in the Kähler potential (4.8). The SUSY breaking by $F^{Z}$ cancels the cosmological constant, implying that $V_{0}(\tau)$ does not play an important role in stabilizing the modulus $\tau$. The $\mathrm{U}(1)_{A}$-charged $\phi_{i}$ are stabilized away from the origin if they obtain tachyonic soft masses and/or sizable $A$-term. For instance, if the $A$-term is small as

$$
\left|A_{\phi}\right| \ll \sqrt{\left|m_{i}^{2}\right|} \sim m_{\mathrm{SUSY}}
$$

the scalar potential has a minimum at

$$
\begin{aligned}
& v_{1}\left(t_{0}\right) \sim\left(\frac{m_{\mathrm{SUSY}} M_{P l}^{n}}{\lambda}\right)^{1 /(n+1)}, \\
& v_{2}\left(t_{0}\right) \sim \frac{A_{\phi} v_{1}\left(t_{0}\right)}{m_{\mathrm{SUSY}}} \ll v_{1}\left(t_{0}\right),
\end{aligned}
$$

while giving a small FI term:

$$
\xi_{\mathrm{FI}} \simeq v_{1}^{2}\left(t_{0}\right) / 2
$$

implying that $\tau$ is fixed at

$$
\langle\tau\rangle \simeq \tau_{0}+\frac{v_{1}^{2}\left(t_{0}\right)}{2 \delta_{\mathrm{GS}} \partial_{\tau}^{2} K_{0}\left(\tau_{0}\right) M_{P l}^{2}} .
$$

On the other hand, in the opposite limit with

$$
\sqrt{\left|m_{i}^{2}\right|} \ll\left|A_{\phi}\right| \sim m_{\mathrm{SUSY}}
$$

the scalar potential has a minimum at

$$
v_{1}\left(t_{0}\right) \simeq \sqrt{n+2} v_{2}\left(t_{0}\right) \sim\left(\frac{m_{\mathrm{SUSY}} M_{P l}^{n}}{\lambda}\right)^{1 /(n+1)},
$$


with a tiny FI term, $\xi_{\mathrm{FI}} \ll v_{1}^{2}\left(t_{0}\right)$. As a result, in both cases, the $\mathrm{QCD}$ axion component and its decay constant are determined as

$$
\begin{aligned}
\frac{1}{8 \pi^{2} \delta_{\mathrm{GS}}} \frac{a\left(t_{0}\right)}{f_{a}\left(t_{0}\right)} & =\frac{\theta_{\mathrm{st}}}{\delta_{\mathrm{GS}}}-\frac{v_{1}^{2}\left(t_{0}\right)}{v^{2}\left(t_{0}\right)} \theta_{1}+(n+2) \frac{v_{2}^{2}\left(t_{0}\right)}{v^{2}\left(t_{0}\right)} \theta_{2} \sim-\frac{v_{1}^{2}\left(t_{0}\right)}{v^{2}\left(t_{0}\right)} \theta_{1}+(n+2) \frac{v_{2}^{2}\left(t_{0}\right)}{v^{2}\left(t_{0}\right)} \theta_{2}, \\
f_{a}\left(t_{0}\right) & =\frac{f_{\mathrm{st}} v\left(t_{0}\right)}{\sqrt{\left(8 \pi^{2} \delta_{\mathrm{GS}} f_{\mathrm{st}}\right)^{2}+v^{2}\left(t_{0}\right)}} \sim\left(\frac{m_{\mathrm{SUSY}} M_{P l}^{n}}{\lambda}\right)^{1 /(n+1)}
\end{aligned}
$$

where

$$
f_{\mathrm{st}} \simeq \frac{\sqrt{2 \partial_{\tau}^{2} K_{0}}}{8 \pi^{2}} M_{P l}, \quad v^{2}=v_{1}^{2}+(n+2)^{2} v_{2}^{2},
$$

and the last equality in (4.20) holds for $v\left(t_{0}\right) \ll 8 \pi^{2} \delta_{\mathrm{GS}} f_{\mathrm{st}}$, i.e. when

$$
m_{\mathrm{SUSY}} \ll 10^{16-2 n}\left(\frac{\lambda}{1.0}\right)\left(\frac{\delta_{\mathrm{GS}}}{10^{-2}}\right)^{n+1} \mathrm{GeV} .
$$

Let us move on to the scalar potential during inflation with the inflaton field

$$
\varphi\left(t_{I}\right)=\operatorname{Im}\left(\Phi\left(t_{I}\right)\right)>M_{P l}
$$

In this period, the inflaton sector generates a large positive vacuum energy

$$
V\left(t_{I}\right)=3 H_{I}^{2} M_{P l}^{2} \simeq\left|F^{X}\right|^{2}=\mu^{2} \varphi^{2}\left(t_{I}\right) .
$$

Note that $V_{0}(\tau)$ in (4.11) is of the order of $H_{I}^{2} M_{P l}^{2}$, and thus becomes important in high scale inflation with $H_{I} \gg m_{3 / 2} \cdot{ }^{7}$ Such a large $V_{0}$ enhances the $\mathrm{U}(1)_{A} D$-term, which can be seen from the minimization condition $\partial_{\tau} V=0$, yielding

$$
g_{A}^{2} D_{A} \simeq \frac{3}{\delta_{\mathrm{GS}} \partial_{\tau}^{2} K_{0}}\left(\frac{\left|\phi_{1}\right|^{2}-(n+2)\left|\phi_{2}\right|^{2}}{\delta_{\mathrm{GS}} M_{P l}^{2}}-\kappa\right) H_{I}^{2},
$$

where $\kappa$ is the coupling between the GS modulus-axion superfield $T$ and the SUSY breaking superfield $X$ in the Kähler potential (4.8), and we have used the relation $\xi_{\mathrm{FI}}=$ $\delta_{\mathrm{GS}} \partial_{\tau} K_{0} M_{P l}^{2}$.

In fact, this expectation value of the $D$-term plays a crucial role for the determination of the vacuum value of $\phi_{i}$ during inflation. For the coupling (4.8), SUSY breaking soft masses of $\phi_{i}$ during inflation are given by

$$
\begin{aligned}
\tilde{m}_{i}^{2} & =m_{i}^{2}+q_{i} g_{A}^{2} D_{A} \\
& \simeq\left(1-\kappa_{i}\right) \frac{\left|F^{X}\right|^{2}}{M_{P l}^{2}}+q_{i} g_{A}^{2} D_{A}=\mathcal{O}\left(\left(1-\kappa_{i}\right) H_{I}^{2}\right)+\mathcal{O}\left(8 \pi^{2} \kappa H_{I}^{2}\right),
\end{aligned}
$$

\footnotetext{
${ }^{7}$ This also implies that the scalar potential of a light modulus can be significantly modified during inflation, which may cause the moduli runaway problem in high scale inflation [77]. In our case, the GS modulus $\tau$ obtains a heavy mass $M_{\tau} \sim \delta_{\mathrm{GS}} M_{P l} \gg H_{I}$ by the $\mathrm{U}(1)_{A} D$-term potential, and thus is free from the runaway problem. For other moduli, if exist, we simply assume that they also have a supersymmetric mass heavy enough to be free from the runaway problem.
} 
where we have used the result (4.24) with $\delta_{\mathrm{GS}}=\mathcal{O}\left(1 / 8 \pi^{2}\right)$. This suggests that, for the parameter region with

$$
\kappa \sim\left(1-\kappa_{i}\right),
$$

which is presumably a natural choice, the soft masses are dominated by the $D$-term contribution, and then the symmetric solution $\phi_{1}=\phi_{2}=0$ can not be a stable solution as $\phi_{i}$ have an opposite sign of $\mathrm{U}(1)_{A}$ charges. We then have

$$
\begin{aligned}
& v_{1}\left(t_{I}\right) \sim\left(\frac{H_{I} M_{P l}^{n}}{\lambda\left|\delta_{\mathrm{GS}}\right|^{1 / 2}}\right)^{1 /(n+1)}, \\
& v_{2}\left(t_{I}\right) \sim \frac{A_{\phi} v_{1}\left(t_{I}\right)}{\left|\delta_{\mathrm{GS}}\right|^{1 / 2} H_{I}},
\end{aligned}
$$

with

$$
\langle\tau\rangle \simeq \tau_{0}+\frac{v_{1}^{2}\left(t_{I}\right)}{2 \delta_{\mathrm{GS}} \partial_{\tau}^{2} K_{0}\left(\tau_{0}\right) M_{P l}^{2}} .
$$

We note that $\left|A_{\phi}\right| \ll H_{I}$ in the chaotic inflation under consideration, because the SUSY breaking field $X$ is odd under $Z_{2}$, and carries a non-zero $R$-charge, which results in $v_{1}\left(t_{I}\right) \gg v_{2}\left(t_{I}\right)$. Then the QCD axion component and its decay constant during inflation are determined as

$$
\begin{aligned}
\frac{1}{8 \pi^{2} \delta_{\mathrm{GS}}} \frac{a\left(t_{I}\right)}{f_{a}\left(t_{I}\right)} & =\frac{\theta_{\mathrm{st}}}{\delta_{\mathrm{GS}}}-\frac{v_{1}^{2}\left(t_{I}\right)}{v^{2}\left(t_{I}\right)} \theta_{1}+(n+2) \frac{v_{2}^{2}\left(t_{I}\right)}{v^{2}\left(t_{I}\right)} \theta_{2} \sim \frac{\theta_{\mathrm{st}}}{\delta_{\mathrm{GS}}}-\theta_{1}, \\
f_{a}\left(t_{I}\right) & \simeq \frac{f_{\mathrm{st}} v_{1}\left(t_{I}\right)}{\sqrt{\left(8 \pi^{2} \delta_{\mathrm{GS}} f_{\mathrm{st}}\right)^{2}+v_{1}^{2}\left(t_{I}\right)}} .
\end{aligned}
$$

As noticed from the discussion in the previous section, a larger axion decay constant during inflation makes it easier to satisfy the constraints on the axion isocurvature perturbation and non-Gaussianity. On the other hand, $f_{a}\left(t_{I}\right)$ in our framework is bounded as

$$
f_{a}\left(t_{I}\right) \sim \operatorname{Min}\left(f_{\mathrm{st}}, v_{i}\left(t_{I}\right)\right)
$$

implying that we need

$$
v_{1}\left(t_{I}\right) \gtrsim f_{\mathrm{st}}=\frac{\sqrt{2 \partial_{\tau}^{2} K_{0}}}{8 \pi^{2}} M_{P l}=\mathcal{O}\left(10^{16}-10^{17}\right) \mathrm{GeV}
$$

to saturate the bound as

$$
f_{a}\left(t_{I}\right) \simeq f_{\text {st. }}
$$

Such a large expectation value of $\mathrm{U}(1)_{A^{-}}$-charged matter fields can be obtained in high scale inflation with

$$
H_{I} \gtrsim 10^{15-2 n} \mathrm{GeV} \times\left(\frac{\lambda}{1.0}\right)\left(\frac{\delta_{\mathrm{GS}}}{10^{-2}}\right)^{n+3 / 2},
$$

which follows from the relation (4.27). The above is indeed the case for $H_{I} \simeq 10^{14} \mathrm{GeV}$ when

$$
n=0 \text { and } \lambda \lesssim 0.1, \quad \text { or } \quad n \geq 1 \text { and } \lambda \lesssim 1 \text {. }
$$


Finally we note that, to restore the PQ symmetry, the modulus coupling to the inflaton sector should be suppressed as

$$
|\kappa| \lesssim \delta_{\mathrm{GS}} \partial_{\tau}^{2} K_{0}\left|1-\kappa_{i}\right|
$$

which means that the GS modulus-axion superfield $T$ is significantly more sequestered from the SUSY breaking in the inflaton sector than the $\mathrm{U}(1)_{A}$-charged matter fields. In addition, we need to arrange the model parameters to make $\tilde{m}_{i}^{2}=m_{i}^{2}+q_{i} g_{A}^{2} D_{A}$ positive for both $\phi_{1}$ and $\phi_{2}$.

\section{Conclusions}

In this paper, we have examined the cosmological constraints on string theoretic QCD axion in the light of the recent PLANCK and BICEP2 results. We were focusing on models with anomalous $\mathrm{U}(1)_{A}$ gauge symmetry, which admit a supersymmetric solution with vanishing Fayet-Illiopoulos $(\mathrm{FI})$ term $\xi_{\mathrm{FI}}=0$, as such models can be realized in many of the known compactified string theories, while being consistent with all the known cosmological constraints for a certain range of model parameters.

If the QCD axion is charged under $\mathrm{U}(1)_{A}$, the axion decay constant is determined essentially by the vacuum expectation values of $\mathrm{U}(1)_{A}$ charged matter fields $\phi$. To have a phenomenologically viable axion scale, the supersymmetric solution $\xi_{\mathrm{FI}}=\phi=0$ should be destabilized by a tachyonic SUSY breaking mass of $\phi$, which would result in an intriguing connection between the axion scale and the SUSY breaking soft masses in the present universe: $f_{a}\left(t_{0}\right) \sim\left(m_{\text {SUSY }} M_{P l}^{n}\right)^{1 /(n+1)}(n \geq 0)$. We note that such models can have rich symmetry breaking patterns during inflation, and therefore allow a certain range of the model parameters compatible with strong cosmological constraints.

If the modulus-axion superfield implementing the Green-Schwarz (GS) anomaly cancellation mechanism is not sequestered from the SUSY breaking by the inflaton sector, the $\mathrm{U}(1)_{A}$-charged matter fields develop a large expectation value $\left\langle\phi\left(t_{I}\right)\right\rangle \sim$ $\left(\sqrt{8 \pi^{2}} H_{I} M_{P l}^{n}\right)^{1 /(n+1)}$ during inflation, due to the tachyonic soft scalar mass $m_{\phi}^{2} \sim-8 \pi^{2} H_{I}^{2}$ induced by the $\mathrm{U}(1)_{A} D$-term. This makes it possible that the model is free from the axion domain wall problem, while satisfying the severe constraints on isocurvature density perturbations for the axion scale and relic abundance depicted in figure 1 . If one allows a fine-tuning of the classical axion misalignment angle $\theta_{0}$, then the axion scale in the range $10^{9} \mathrm{GeV}<f_{a}\left(t_{0}\right)<5 \times 10^{13} \mathrm{GeV}$ is cosmologically viable for a reasonable choice of the model parameters. On the other hand, for $\theta_{0}=\mathcal{O}(1)$, the allowed range is reduced to $10^{9} \mathrm{GeV}<f_{a}\left(t_{0}\right)<10^{11} \mathrm{GeV}$, with the relic axions composing up to $0.1-10 \%$ of the total dark matter energy density.

On the other hand, if the dilaton-axion superfield for the GS mechanism is sequestered from the SUSY breaking by the inflaton sector, it is possible that the PQ symmetry is restored during inflation with $\left\langle\phi\left(t_{I}\right)\right\rangle=0$. Such scenario is obviously free from the isocurvature constraint, but is subject to the domain-wall constraint $N_{\mathrm{DW}}=1$. Furthermore, if one adopts the recent numerical simulation for the axion production by the annihilations 
of axionic stings and domain walls for the case of $N_{\mathrm{DW}}=1$, one finds that only a narrow range of the axion decay constant, $10^{9} \mathrm{GeV}<f_{a}\left(t_{0}\right)<$ (a few) $\times 10^{10} \mathrm{GeV}$, is allowed.

Open Access. This article is distributed under the terms of the Creative Commons Attribution License (CC-BY 4.0), which permits any use, distribution and reproduction in any medium, provided the original author(s) and source are credited.

\section{References}

[1] J.E. Kim and G. Carosi, Axions and the strong CP problem, Rev. Mod. Phys. 82 (2010) 557 [arXiv:0807.3125] [INSPIRE].

[2] A. Ringwald, Exploring the role of axions and other WISPs in the dark universe, Phys. Dark Univ. 1 (2012) 116 [arXiv:1210.5081] [INSPIRE].

[3] M. Kawasaki and K. Nakayama, Axions: theory and cosmological role, Ann. Rev. Nucl. Part. Sci. 63 (2013) 69 [arXiv:1301.1123] [InSPIRE].

[4] R.D. Peccei and H.R. Quinn, CP conservation in the presence of instantons, Phys. Rev. Lett. 38 (1977) 1440 [INSPIRE].

[5] R.D. Peccei and H.R. Quinn, Constraints imposed by CP conservation in the presence of instantons, Phys. Rev. D 16 (1977) 1791 [InSPIRE].

[6] S. Weinberg, A new light boson?, Phys. Rev. Lett. 40 (1978) 223 [InSPIRE].

[7] F. Wilczek, Problem of strong $p$ and $t$ invariance in the presence of instantons, Phys. Rev. Lett. 40 (1978) 279 [INSPIRE].

[8] J.E. Kim, Weak interaction singlet and strong CP invariance, Phys. Rev. Lett. 43 (1979) 103 [INSPIRE].

[9] M.A. Shifman, A.I. Vainshtein and V.I. Zakharov, Can confinement ensure natural CP invariance of strong interactions?, Nucl. Phys. B 166 (1980) 493 [INSPIRE].

[10] M. Dine, W. Fischler and M. Srednicki, A simple solution to the strong CP problem with a harmless axion, Phys. Lett. B 104 (1981) 199 [INSPIRE].

[11] A.R. Zhitnitsky, On possible suppression of the axion hadron interactions (in Russian), Sov. J. Nucl. Phys. 31 (1980) 260 [Yad. Fiz. 31 (1980) 497] [INSPIRE].

[12] L.F. Abbott and M.B. Wise, Wormholes and global symmetries, Nucl. Phys. B 325 (1989) 687 [inSPIRE].

[13] S.R. Coleman and K.-M. Lee, Wormholes made without massless matter fields, Nucl. Phys. B 329 (1990) 387 [InSPIRE].

[14] R. Kallosh, A.D. Linde, D.A. Linde and L. Susskind, Gravity and global symmetries, Phys. Rev. D 52 (1995) 912 [hep-th/9502069] [InSPIRE].

[15] T. Banks and N. Seiberg, Symmetries and strings in field theory and gravity, Phys. Rev. D 83 (2011) 084019 [arXiv:1011.5120] [inSPIRE].

[16] S.M. Barr and D. Seckel, Planck scale corrections to axion models, Phys. Rev. D 46 (1992) 539 [INSPIRE].

[17] M. Kamionkowski and J. March-Russell, Planck scale physics and the Peccei-Quinn mechanism, Phys. Lett. B 282 (1992) 137 [hep-th/9202003] [INSPIRE]. 
[18] R. Holman et al., Solutions to the strong CP problem in a world with gravity, Phys. Lett. B 282 (1992) 132 [hep-ph/9203206] [INSPIRE].

[19] E. Witten, Some properties of O(32) superstrings, Phys. Lett. B 149 (1984) 351 [InSPIRE].

[20] M.B. Green, J.H. Schwarz and E. Witten, Superstring theory, vol. 2, Cambridge University Press, Cambridge U.K. (1987).

[21] L.E. Ibanez and A.M. Uranga, String theory and particle physics: an introduction to string phenomenology, Cambridge University Press, Cambridge U.K. (2012).

[22] K. Choi and J.E. Kim, Harmful axions in superstring models, Phys. Lett. B 154 (1985) 393 [Erratum ibid. B 156 (1985) 452] [INSPIRE].

[23] K. Choi and J.E. Kim, Compactification and axions in $E_{8} \times E_{8}^{\prime}$ superstring models, Phys. Lett. B 165 (1985) 71 [INSPIRE].

[24] P. Svrček and E. Witten, Axions in string theory, JHEP 06 (2006) 051 [hep-th/0605206] [INSPIRE].

[25] T. Banks and M. Dine, Couplings and scales in strongly coupled heterotic string theory, Nucl. Phys. B 479 (1996) 173 [hep-th/9605136] [INSPIRE].

[26] K. Choi, Axions and the strong CP problem in M-theory, Phys. Rev. D 56 (1997) 6588 [hep-th/9706171] [INSPIRE].

[27] J. Preskill, M.B. Wise and F. Wilczek, Cosmology of the invisible axion, Phys. Lett. B 120 (1983) 127 [inSPIRE].

[28] L.F. Abbott and P. Sikivie, A cosmological bound on the invisible axion, Phys. Lett. B 120 (1983) 133 [INSPIRE].

[29] M. Dine and W. Fischler, The not so harmless axion, Phys. Lett. B 120 (1983) 137 [INSPIRE].

[30] M. Axenides, R.H. Brandenberger and M.S. Turner, Development of axion perturbations in an axion dominated universe, Phys. Lett. B 126 (1983) 178 [INSPIRE].

[31] D. Seckel and M.S. Turner, Isothermal density perturbations in an axion dominated inflationary universe, Phys. Rev. D 32 (1985) 3178 [INSPIRE].

[32] A.D. Linde, Generation of isothermal density perturbations in the inflationary universe, Phys. Lett. B 158 (1985) 375 [InSPIRE].

[33] D.H. Lyth, A limit on the inflationary energy density from axion isocurvature fluctuations, Phys. Lett. B 236 (1990) 408 [INSPIRE].

[34] M.S. Turner and F. Wilczek, Inflationary axion cosmology, Phys. Rev. Lett. 66 (1991) 5 [INSPIRE].

[35] P. Fox, A. Pierce and S.D. Thomas, Probing a QCD string axion with precision cosmological measurements, hep-th/0409059 [INSPIRE].

[36] K.J. Mack and P.J. Steinhardt, Cosmological problems with multiple axion-like fields, JCAP 05 (2011) 001 [arXiv:0911.0418] [INSPIRE].

[37] K.J. Mack, Axions, inflation and the anthropic principle, JCAP 07 (2011) 021 [arXiv: 0911.0421] [INSPIRE].

[38] M.B. Green and J.H. Schwarz, Anomaly cancellation in supersymmetric D $=10$ gauge theory and superstring theory, Phys. Lett. B 149 (1984) 117 [INSPIRE]. 
[39] S.M. Barr, Harmless axions in superstring theories, Phys. Lett. B 158 (1985) 397 [INSPIRE].

[40] J.E. Kim, The strong CP problem in orbifold compactifications and an $\mathrm{SU}(3) \times \mathrm{SU}(2) \times \mathrm{U}(1)^{n}$ model, Phys. Lett. B $207(1988) 434$ [INSPIRE].

[41] K. Choi, K.S. Jeong, K.-I. Okumura and M. Yamaguchi, Mixed mediation of supersymmetry breaking with anomalous U(1) gauge symmetry, JHEP 06 (2011) 049 [arXiv:1104.3274] [INSPIRE].

[42] G. Honecker and W. Staessens, On axionic dark matter in type IIA string theory, Fortsch. Phys. 62 (2014) 115 [arXiv:1312.4517] [INSPIRE].

[43] M. Cvetič, G. Shiu and A.M. Uranga, Chiral four-dimensional $N=1$ supersymmetric type $2 A$ orientifolds from intersecting D6 branes, Nucl. Phys. B 615 (2001) 3 [hep-th/0107166] [INSPIRE].

[44] D. Cremades, L.E. Ibáñez and F. Marchesano, SUSY quivers, intersecting branes and the modest hierarchy problem, JHEP 07 (2002) 009 [hep-th/0201205] [INSPIRE].

[45] G. Honecker and T. Ott, Getting just the supersymmetric Standard Model at intersecting branes on the $Z_{6}$ orientifold, Phys. Rev. D 70 (2004) 126010 [Erratum ibid. D 71 (2005) 069902] [hep-th/0404055] [INSPIRE].

[46] G. Villadoro and F. Zwirner, D terms from D-branes, gauge invariance and moduli stabilization in flux compactifications, JHEP 03 (2006) 087 [hep-th/0602120] [INSPIRE].

[47] F. Gmeiner and G. Honecker, Millions of Standard Models on Z6 ?, JHEP 07 (2008) 052 [arXiv:0806.3039] [INSPIRE].

[48] R. Blumenhagen, G. Honecker and T. Weigand, Loop-corrected compactifications of the heterotic string with line bundles, JHEP 06 (2005) 020 [hep-th/0504232] [INSPIRE].

[49] R. Blumenhagen, G. Honecker and T. Weigand, Supersymmetric (non-)Abelian bundles in the type I and $\mathrm{SO}(32)$ heterotic string, JHEP 08 (2005) 009 [hep-th/0507041] [INSPIRE].

[50] L.B. Anderson, J. Gray, A. Lukas and B. Ovrut, Stability walls in heterotic theories, JHEP 09 (2009) 026 [arXiv:0905.1748] [INSPIRE].

[51] H. Murayama, H. Suzuki and T. Yanagida, Radiative breaking of Peccei-Quinn symmetry at the intermediate mass scale, Phys. Lett. B 291 (1992) 418 [INSPIRE].

[52] K. Choi, E.J. Chun and J.E. Kim, Cosmological implications of radiatively generated axion scale, Phys. Lett. B 403 (1997) 209 [hep-ph/9608222] [INSPIRE].

[53] BICEP2 collaboration, P.A.R. Ade et al., Detection of B-mode polarization at degree angular scales by BICEP2, Phys. Rev. Lett. 112 (2014) 241101 [arXiv: 1403.3985] [INSPIRE].

[54] D.J.E. Marsh, D. Grin, R. Hlozek and P.G. Ferreira, Tensor detection severely constrains axion dark matter, Phys. Rev. Lett. 113 (2014) 011801 [arXiv: 1403.4216] [INSPIRE].

[55] L. Visinelli and P. Gondolo, Axion cold dark matter in view of BICEP2 results, Phys. Rev. Lett. 113 (2014) 011802 [arXiv: 1403.4594] [INSPIRE].

[56] A.G. Dias, A.C.B. Machado, C.C. Nishi, A. Ringwald and P. Vaudrevange, The quest for an intermediate-scale accidental axion and further ALPs, JHEP 06 (2014) 037 [arXiv: 1403.5760] [INSPIRE].

[57] Planck collaboration, P.A.R. Ade et al., Planck 2013 results. XXII. Constraints on inflation, arXiv: 1303.5082 [INSPIRE]. 
[58] K.S. Jeong and F. Takahashi, Suppressing isocurvature perturbations of QCD axion dark matter, Phys. Lett. B 727 (2013) 448 [arXiv:1304.8131] [InSPIRE].

[59] T. Higaki, K.S. Jeong and F. Takahashi, Solving the tension between high-scale inflation and axion isocurvature perturbations, Phys. Lett. B 734 (2014) 21 [arXiv:1403.4186] [INSPIRE].

[60] T. Hiramatsu, M. Kawasaki, K. Saikawa and T. Sekiguchi, Production of dark matter axions from collapse of string-wall systems, Phys. Rev. D 85 (2012) 105020 [Erratum ibid. D 86 (2012) 089902] [arXiv:1202.5851] [INSPIRE].

[61] T. Hiramatsu, M. Kawasaki, K. Saikawa and T. Sekiguchi, Axion cosmology with long-lived domain walls, JCAP 01 (2013) 001 [arXiv:1207.3166] [INSPIRE].

[62] L.J. Hall, Y. Nomura and S. Shirai, Grand unification, axion and inflation in intermediate scale supersymmetry, JHEP 06 (2014) 137 [arXiv:1403.8138] [INSPIRE].

[63] K.-W. Choi, A QCD axion from higher dimensional gauge field, Phys. Rev. Lett. 92 (2004) 101602 [hep-ph/0308024] [InSPIRE].

[64] I.-W. Kim and J.E. Kim, Modification of decay constants of superstring axions: effects of flux compactification and axion mixing, Phys. Lett. B 639 (2006) 342 [hep-th/0605256] [INSPIRE].

[65] M. Cicoli et al., Explicit de Sitter flux vacua for global string models with chiral matter, JHEP 05 (2014) 001 [arXiv:1312.0014] [INSPIRE].

[66] R. Allahverdi, M. Cicoli, B. Dutta and K. Sinha, Correlation between dark matter and dark radiation in string compactifications, arXiv:1401.4364 [INSPIRE].

[67] Y. Kawamura, Model independent analysis of soft masses in heterotic string models with anomalous U(1) symmetry, Phys. Lett. B 446 (1999) 228 [hep-ph/9811312] [INSPIRE].

[68] K. Choi and K.S. Jeong, Supersymmetry breaking and moduli stabilization with anomalous U(1) gauge symmetry, JHEP 08 (2006) 007 [hep-th/0605108] [INSPIRE].

[69] M.S. Turner, Cosmic and local mass density of invisible axions, Phys. Rev. D 33 (1986) 889 [INSPIRE].

[70] D.H. Lyth, Axions and inflation: sitting in the vacuum, Phys. Rev. D 45 (1992) 3394 [INSPIRE].

[71] K.J. Bae, J.-H. Huh and J.E. Kim, Update of axion CDM energy, JCAP 09 (2008) 005 [arXiv:0806.0497] [INSPIRE].

[72] L. Visinelli and P. Gondolo, Dark matter axions revisited, Phys. Rev. D 80 (2009) 035024 [arXiv:0903.4377] [INSPIRE].

[73] T. Kobayashi, R. Kurematsu and F. Takahashi, Isocurvature constraints and anharmonic effects on QCD axion dark matter, JCAP 09 (2013) 032 [arXiv: 1304.0922] [INSPIRE].

[74] M. Kawasaki, K. Nakayama, T. Sekiguchi, T. Suyama and F. Takahashi, Non-Gaussianity from isocurvature perturbations, JCAP 11 (2008) 019 [arXiv:0808.0009] [INSPIRE].

[75] A.D. Linde, Axions in inflationary cosmology, Phys. Lett. B 259 (1991) 38 [InSPIRE].

[76] M. Kawasaki, M. Yamaguchi and T. Yanagida, Natural chaotic inflation in supergravity, Phys. Rev. Lett. 85 (2000) 3572 [hep-ph/0004243] [INSPIRE].

[77] R. Kallosh and A.D. Linde, Landscape, the scale of SUSY breaking and inflation, JHEP 12 (2004) 004 [hep-th/0411011] [INSPIRE]. 